\title{
Caracterisation et traitement de boues de dragages contenant des polluants métalliques : exemple d'une boue de canal.
}

\author{
Franck Marot \\ Etudiant en thèse, Ademe/BRGM, LMFGC, Université du Havre
}

\author{
Pierre Conil \\ Ingénieur de recherche, $B R G M$ Oriéans
}

Blandine Clozel

Ingénieur de recherche, $B R G M$ Orléans

\author{
Daniel Levacher \\ Professeur des universités, LMFGC, Université du Havre
}

\section{Résumé}

Les travaux présentés s'inscrivent dans une étude dont l'objectif est de déterminer les possibilités de décontamination des vases contenant des polluants métalliques. Les échantillons utilisés correspondent à une boue fortement polluée (certains métaux atteignent des teneurs de l'ordre du pour cent), prélevée dans un canal de la région Nord - Pas de Calais. Une caractérisation physique et chimique de ces produits a tout d'abord été entreprise, afin d'orienter, dans un deuxième temps, les essais de traitement. Les formes chimiques sous lesquelles s'expriment les éléments métalliques s'avèrent très nombreuses et souvent délicates à déterminer. Malgré cette complexité, les essais préliminaires de décontamination ont permis de définir l'ébauche d'un procédé de traitement. Ils permettent de diviser les teneurs par 6 pour le zinc, par 11 pour le plomb et par 14 pour le cadmium, sur une masse représentant un tiers de la boue brute. La poursuite de l'étude vise à améliorer le rendement massique de l'opération. L'application, sur d'autres boues, de l'ensemble de la démarche est également envisagée.

\section{Introduction}

L'entretien des voies navigables génère des volumes de vases considérables. Quarante millions de mètres cube sont dragués annuellement dans les estuaires français. Pour les canaux, la seule région Nord - Pas de Calais totalise un million de mètres cube par an de boues de curage. Pourtant, en plus de ces problèmes de volume, deux paramètres particuliers viennent renforcer les difficultés rencontrées avec ces produits :

- Un comportement mécanique défavorable. En effet, parfois plastiques, souvent liquides, les vases sont surtout thixotropiques, c'est à dire qu'elles perdent leur résistance mécanique dès qu'elles sont sollicitées. Cette particularité provient en partie des fortes teneurs en eau et des granulométries excessivement fines de ces boues. Cette propriété entraine de sérieux problèmes tant pour la manutention (lors du dragage, du transport, ...) que pour le stockage (en particulier problème de stabilité mécanique et de réutilisation à moyen ou long terme des sites de stockage).

- Des contaminations éventuelles par des polluants. Avec de fortes proportions en argile et en matières organiques, les vases sont susceptibles de piéger de nombreux polluants. Ainsi, selon leur environnement, elles peuvent parfois être très largement contaminées. Lorsque cela se produit, la problématique du devenir des matériaux de dragage devient alors encore plus complexe en raison des contraintes plus rigides sur la mise en stockage. 
Afin de pouvoir définir et évaluer des procédés permettant de décontaminer des vases, lorsque celles-ci contiennent des polluants métalliques, il s'avère nécessaire de procéder à une étude comportant la succession d'étapes suivante : caractérisation des matériaux et des contaminants, essais préliminaires de traitement, essais d'optimisation et évaluation technico-économique. La présentation ci-après a pour objet de décrire, à partir de l'exemple d'une boue de canal, les deux premières étapes de cette méthodologie:

- caractérisation. Outre la quantification des contaminations, cette étape vise ̀̀ déterminer les caractéristiques physiques du matériau é la nature de ses constituants. Un effort particulier est entrepris afin de localiser les polluants métalliques et d'essayer de définir les formes sous lesquelles ils s'expriment. En effet ces informations sont très précieuses pour le choix des techniques de traitement et l'interprétation de leurs résultats, lors des étapes suivantes.

- essais préliminaires de traitement. Ces tests ont pour objectif une première évaluation des techniques de traitements envisageables, sélectionnées à partir des résultats de l'étape précédente.

\section{Caractérisation de la vase}

Plusieurs prélèvements réalisés sur un site d'un canal de la région Nord - Pas de Calais ont permis, après mélange et homogénéisation, de constituer un lot d'environ $200 \mathrm{~kg}$ de boues humides représentant les vases "moyennes" accumulées en ce lieu.

\subsection{Caractéristiques physiques}

\subsubsection{Granulométrie}

La figure 1 présente les résultats de l'analyse granulométrique de ces boues. Elle montre que $80 \%$ des particules ont un diamètre inférieur à $90 \mu \mathrm{m}\left(\mathrm{d}_{80}\right.$ de $\left.90 \mu \mathrm{m}\right)$ et $12 \%$ sont inférieures à $10 \mu \mathrm{m}$. Ces paramètres caractérisent une boue assez grossière. A titre de comparaison, les vases d'estuaires contiennent généralement plus de $50 \%$ de particules inférieures à $10 \mu \mathrm{m}$. (Roger B., 1988 / Sanchez M., 1993)

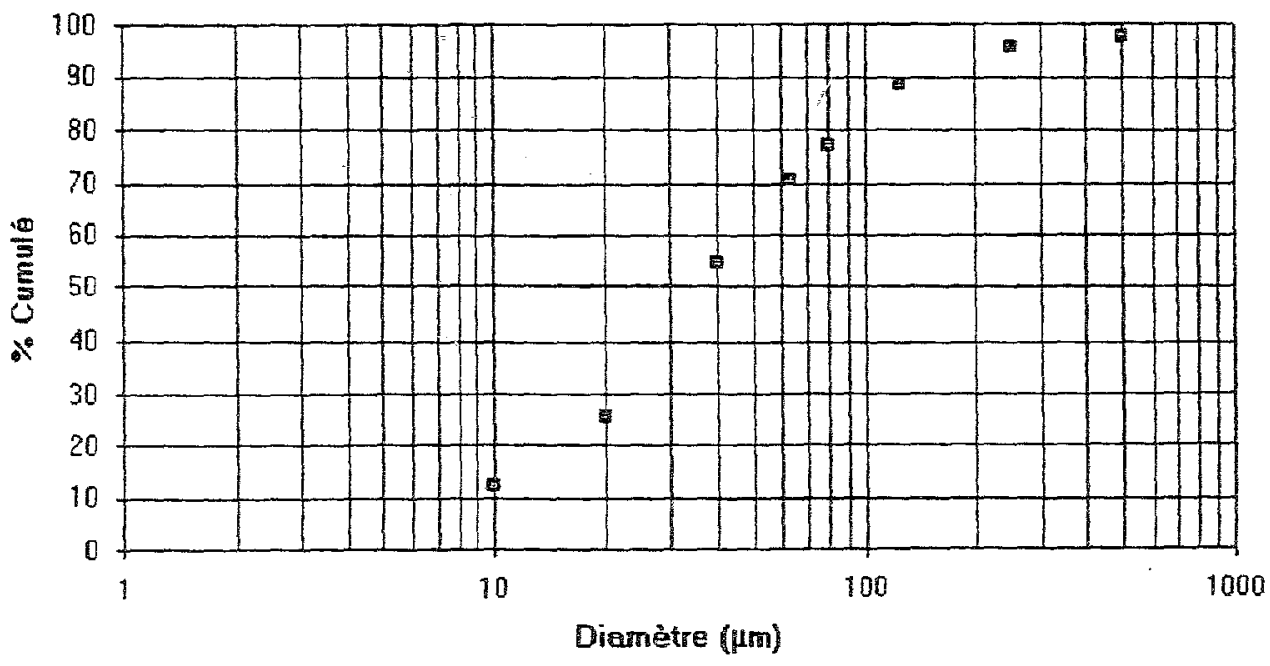

figure 1 - Courbe granulométrique de la vase prélevée 


\subsubsection{Perméabilité}

Des mesures de sédimentation à intervalle de temps croissant ont été effectuées sur deux colonnes de $1.10 \mathrm{~m}$ et $1.60 \mathrm{~m}$ de hauteur. La concentration initiale identique dans les deux cas était de $160 \mathrm{~kg} / \mathrm{m}^{3}$. L'essai a duré 16 jours. Les courbes de sédimentation permettent de déterminer la perméabilité de ces boues en fonction de la concentration (méthode de Kynch). Les deux essais conduisent à des perméabilités identiques (figure 2). Deux comportements se succèdent, avec tout d'abord un domaine linéaire, où le coefficient de perméabilité varie de $2.10^{-4}$ à $5.10^{-7} \mathrm{~m} / \mathrm{s}$ pour des concentrations comprises entre 150 et $350 \mathrm{~g} / \mathrm{l}$. Puis un domaine non linéaire, au-delà de $350 \mathrm{~g} / \mathrm{l}$, montre que la perméabilité diminue alors très rapidement lorsque la concentration augmente. Les résultats obtenus n'ont toutefois qu'une valeur indicative car des effets de parois peuvent jouer un rôle important en ralentissant la sédimentation et le tassement des boues. L'inconvénient des mesures en laboratoire est d'opérer sur des échantillons trop petits pour fournir une représentation parfaitement extrapolable de la perméabilité d'un matériau. "Les perméabilités mesurées en laboratoire sont toujours inférieures à celles mesurées in-situ." (Schlosser F., 1988)

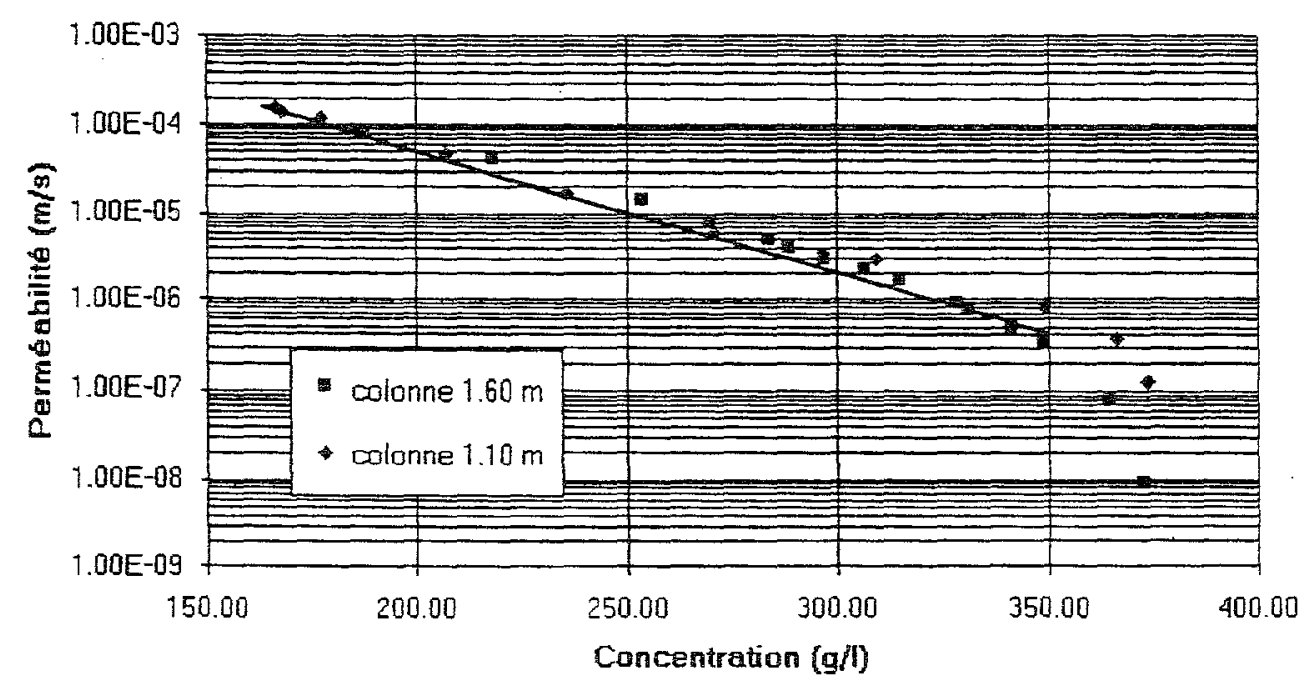

figure 2 - Evolution de la perméabilité en fonction de la concentration solide

\subsection{Caractéristiques chimiques et minéralogiques}

L'analyse chimique de ces produits a révélé des teneurs élevées pour certains métaux : zinc (11 $000 \mathrm{ppm}$ ), plomb (5000 ppm), cadmium (600 ppm) et mercure (29 ppm). A titre purement indicatif ces teneurs sont, dans le tableau 1, présentées en parallèle avec la norme française d'épandage des boues de station d'épuration sur les sols agricoles. Cette norme n'est en effer pas juridiquement applicable aux boues de dragage, pour lesquelles il n'existe pas actuellement de réglementation portant sur des teneurs maximales. La concentration en cadmium est 15 fois supérieure à la valeur limite de cette norme.

\begin{tabular}{|c|c|c|c|c|}
\hline Teneur (ppm) & Zinc & Plomb & Cadmium & Mercure \\
\hline Boue de canal & 11000 & 5000 & 600 & 29 \\
\hline Norme française & 6000 & 1600 & 40 & 10 \\
\hline
\end{tabular}

tableau 1 - Comparaison entre les teneurs de la boue prélevée et la norme française d'épandage des boues de station d'épuration sur des sols agricoles 
Une analyse chimique des métaux en fonction de la taille des particules (analyse granulochimique) a montré que toutes les fractions granulométriques sont polluées. Il y a cependant une accentuation notable des teneurs sur les fines particules $(<20 \mu \mathrm{m})$ et sur les grosses $(>80 \mu \mathrm{m})$. Cette forte contamination des fines n'est pas surprenante. En effet celles-ci contiennent des argiles et de la matière organique, les deux ayant une forte capacité de fixation de polluants. Pour les grosses particules, un encroûtement de grains exempts de polluant par des fines polluées pouvait expliquer une telle distribution granulochimique. L'observation minéralogique ainsi qu'une comparaison entre les teneurs des grosses particules avant et après un décapage mécanique (par attrition) de leurs surfaces ont permis d'éliminer cette hypothèse. La teneur élevée en contaminants dans la tranche granulométrique $>80 \mu \mathrm{m}$ serait donc liée à une distribution dans la masse des grains et non à leur surface.

$\mathrm{La}$ caractérisation des phases majoritaires par diffractométrie des rayons $X$ a montré que le quartz représente près de la moitié de la boue brute. Viennent ensuite la calcite $(20 \%)$ et les feldspaths $(6 \%)$. La fraction argileuse, qui représente environ $30 \%$ de cette boue est constituée de smectite $(70 \%)$, de kaolinite $(23 \%)$ et d'illite libre $(7 \%)$. La chlorite est présente à l'état de trace. Lors de cette première caractérisation, aucun minéral porteur de la pollution n'a été identifié. Cela n'est pas très étonnant, puisque les limites de détection sont de quelques pourcent, alors que les teneurs atteignent au plus $1 \%$ pour le zinc. Ainsi, on peut supposer que la pollution est : "

- soit, très largement distribuée, mais présente à des teneurs faibles et non exprimée sous forme propre (exemples : adsorption ou substitution dans les argiles, sur des oxyhydroxydes de fer ou de manganèse,...),

- soit, très ponctuelle, avec des teneurs élevées, s'exprimant probablement sous forme propre mais non détectée car en trop faible proportion (exemple : minéraux propres de zinc, de plomb,...$)$.

Dans les deux cas, il est nécessaire d'utiliser d'autres outils analytiques pour préciser cette caractérisation. Des analyses ponctuelles, réalisées au MEB (microscope électronique à balayage) et à la microsonde électronique, ont permis d'identifier au moins trois types de phases porteuses d'éléments métalliques:

- D'une part, nous observens la présence de minéraux sulfurés comme la blende (ou sphalérite) $(\mathrm{ZnS})$, la galène $(\mathrm{PbS})$ ou encore la pyrite $\left(\mathrm{FeS}_{2}\right)$. Ces sulfures se présentent soit sous la forme de grains à cassure nette constitués d'un seul minéral, soit sous la forme d'associations polysulfurées. La forme de grains, la nature des phases et les assemblages polymétalliques typiques, indiquent que ces sulfures sont très probablement du minerai dispersé dans la boue.

- D'autre part, nous identifions des fragments de scorie vitreuse (verre silicocalcique à inclusions riches en $\mathrm{Fe}, \mathrm{Zn}, \mathrm{Cu}, \mathrm{Sb}, \mathrm{Co}, \mathrm{Ni}$ ) ou des billes de $\mathrm{Pb}$ métal. Ces phases n'ont pas d'équivalents naturels et sont typiques d'une activité métallurgique.

- Enfin une troisième catégorie de phases porteuses est identifiée. Il s'agit par exemple de petits grains arrondis de taille micronique, dispersés ou en encroûtement, présentant du soufre associé à $\mathrm{Pb}, \mathrm{Zn}$, Fe et parfois $\mathrm{Cd}$ et As. Ces grains se sont formés au sein du sédiment (néoformation) et pourraient être le fruit d'une activité bactérienne.

D'autres grains constitués d'oxyhydroxydes de manganèse présentant des teneurs ponctuelles élevées en $\mathrm{Zn}(13 \%)$ et $\mathrm{Pb}(21 \%)$ ont aussi été identifiés (phénomène d'adsorption et/ou de coprécipitation)

Ces analyses permettent de déterminer les origines les plus probables de la pollution, en précisant les principaux cheminements qui ont conduits les métaux au sein de la boue. Comptetenu du lieu de prélèvement, situé à proximité d"un complexe métallurgique, les deux premiers 
types de phases porteuses d'éléments métalliques (minerai et résidu métallurgique) sont ce que nous pourrions appeler des phases "importées", issues directement de l'activité humaine. Ils se distinguent en cela des autres phases qui sont plutôt le fruit de transformations qui se sont déroulées dans la boue à partir de rejets occasionnés par cette activité industrielle.

\subsection{Essais de comportement}

Les caractérisations précédentes ont montré la grande diversité des formes chimiques sous lesquelles se trouvent les métaux. Il n'est donc pas facile d'orienter les essais de décontamination en fondant quelques espoirs sur un procédé particulier. Cependant, il n'est pas exclu que des comportements similaires puissent exister entre ces différentes formes de polluants lors de traitements chimiques ou physiques. Des essais préliminaires ont donc été réalisés.

\subsubsection{Lixiviation}

L'objectif de ces tests est de déterminer le comportement des constituants du matériau, et en particulier des métaux, en milieu acide. Pour cela des essais de lixiviation (mise en solution) en réacteur agité, à pH stabilisé et contrôlé par des ajouts d'acide sulfurique, ont été réalisés. L'influence de trois niveaux d'acidité du milieu, $\mathrm{pH}=5,3$ et 1 , a été testée sur trois échantillons distincts. L'analyse des filtrats montre qu'une très faible proportion des éléments métalliques passe en solution, même en milieu très acide $(\mathrm{pH}=1)$. Ces résultats indiquent qu'un traitement par lixiviation à l'acide sulfurique ne serait pas efficace sur ces vases.

D'autre part, des sulfures ont été observés dans ces matériaux, lors de la phase de caractérisation. Or, au cours d'un stockage à l'air libre de ces vases, il est possible qu'une légère acidification se produise, par oxydation des sulfures, réaction qui engendre de l'acide sulfurique. Il est possible de déduire des tests de lixiviation réalisés que ce phénomène (s'il se déclenche) n'entraînera pas pour autant de relargage significatif de métaux

\subsubsection{Séparation densimétrique}

Afin de connaître la répartition des métaux entre les différentes fractions densimétriques, des séparations par liqueurs denses ont été effectuées. Cependant, à cause de la viscosité des liquides organiques utilisés, cette technique n'est pas envisageable sur des particules inférieures à $20 \mu \mathrm{m}$, et reste délicate jusqu'à $40 \mu \mathrm{m}$. Ainsi, les résultats qui suivent ne concernent que la fraction supérieure à $40 \mu \mathrm{m}$, soit seulement $30 \%$ de la boue brute. Deux coupures ont été choisies. Une première coupure, à une densité de 2 , permet d'isoler les constituants organiques de cette boue, dont les affinités avec les métaux sont bien connues. (Reuter et Perdue 1977, Davis 1984, Etcheber 1983...). La deuxième coupure, à une densité de 2.77 , permet de séparer les particules lourdes telles que les sulfures, des principaux minéraux du sédiment (quartz, calcite, argiles, ...).

Les analyses chimiques ont montré une forte présence métallique sur la fraction lourde. En effet celle-ci, qui ne représente que $2 \%$ de la masse, concentre $85 \%$ de l'argent, $58 \%$ du plomb, $40 \%$ de l'antimoine, $27 \%$ du cadmium et $20 \%$ de l'étain. Seul le zinc a une teneur quasi constante sur toutes les fractions. On remarque d'autre part que la fraction de densité inférieure à 2 concentre peu de métaux. Les complexations métaux - matière organique semblent donc jouer un rôle mineur. Quant à la fraction de densité intermédiaire, elle s'avère être nettement moins polluée. En conclusion, la séparation densimétrique semble justifiée dans un programme 
de traitement. L'applicabilité de cette technique est à étudier dans le cadre de séparation mécanique (table à secousses) et sur l'ensemble de la boue brute.

\subsubsection{Séparation magnétique}

La susceptibilité magnétique de ces boues est faible. La séparation en voie humide a conduit à une récupération massique de $2.6 \%$ pour une intensité de 25 ampères. Cette fraction magnétique concentre $15 \%$ du fer, $11 \%$ du chromêet du cuivre, et 5 à $6 \%$ du nickel, du zinc, du plomb, de l'étain, de l'antimoine et de l'argent. Ces valeurs sont relativement faibles, malgré un champ magnétique élevé. Le stérile de l'opération qui représente plus de $97 \%$ de la masse initiale, reste fortement pollué. Ce type de traitement n'apporte donc pas de solution efficace.

\section{Essais préliminaires de décontamination}

La phase de caractérisation a montré que les principaux métaux s'expriment sous des formes chimiques très variées. Les formes sulfurées et oxydées semblent cependant être les plus fréquentes. Pour ces dernières, des essais de flottation, faisant appel aux propriétés de surface des particules, peuvent être particulièrement adaptés.

\subsection{Traitement physico-chimique par flottation}

La flottation permet d'effectuer des séparations de solides en utilisant les différences de propriétés de surface de ces solides. La première étape de cette opération est le conditionnement. II consiste à introduire des réactifs (collecteurs, activants, déprimants...) dont le rôle est de rendre hydrophobe la surface des minéraux que l'on souhaite isoler. La pulpe ainsi conditionnée est alors introduite dans des cellules de flottation. Ce sont des réacteurs munis d'agitateurs et générant des bulles d'air au sein de la suspension. Ces dernières vont se fixer sur les particules dont la surface est hydrophobe. Sous l'action de la poussée d'Archimède, l'ensemble particules + bulles flotte à la surface du réacteur en formant une écume qui est stabilisée par l'ajout d'un moussant. L'écume enrichie en phase solide est alors enlevée de la cellule pour former le concentré de flottation.

Ce traitement, avec différentes conditions opératoires, a été appliqué aux boues après que celles-ci aient été débarrassées des fractions granulométriques fines et grossières, qui pertuberaient la séparation. La caractérisation précédente a orienté le choix des réactifs. Les résultats obtenus sont présentés-dans le tableau 2 et s'avèrent assez encourageants. Cette opération a permis de récupérer 80 à $90 \%$ des principaux polluants sur $40 \%$ de la masse initiale.

\begin{tabular}{|c|c|c|c|c|c|c|c|}
\hline \multirow[t]{2}{*}{ Flottation } & \multirow{2}{*}{$\begin{array}{c}\% \\
\text { Poids }\end{array}$} & \multicolumn{2}{|c|}{$\mathrm{Zn}$} & \multicolumn{2}{|c|}{$\mathrm{Pb}$} & \multicolumn{2}{|c|}{$\mathrm{Cd}$} \\
\hline & & Ten.(ppm) & Réc. $(\%)$ & Ten.(ppm) & Réc. $(\%)$ & Ten.(ppm) & Réc. $(\%)$ \\
\hline Flottant & 38.60 & 16682 & 79.18 & 5774 & 78.00 & 1238 & 91.00 \\
\hline Non-fottant & 61.40 & 2757 & 20.82 & 1024 & 22.00 & 077 & 9.00 \\
\hline Boue initiale calcul. & 100.00 & 8132 & 100.00 & 2858 & 100.00 & 525 & 100.00 \\
\hline Boue initiale analys. & & 8059 & & 3255 & & 525 & \\
\hline
\end{tabular}

tableau 2 - Bilan de la flottation

Les teneurs en contaminants du non-flottant sont nettement diminuées. Afin de poursuivre la décontamination, nous avons cherché à caractériser cette poliution résiduelle. Pour cela, nous avons effectué des séparations densimétriques. 


\subsection{Séparation densimétrique sur le stérile de flottation}

Deux coupures densimétriques, à 2.00 et 2.77 comme lors des essais de caractérisation, ont été réalisées sur le stérile de flottation. Les résultats sont présentés dans le tableau 3.

On observe une importante diminution des teneurs de la fraction intermédiaire. Le plomb voit sa concentration réduite de moitié et, pour le cadmium et le zinc, elle est divisée par 1.5. Cette baisse des teneurs est d'autant plus remarquable que les proportions massiques des fractions lourdes et légères sont extrêmement faibles (inférieures à $0.5 \%$ ). Une étape similaire, de séparation densimétrique, serait donc fort intéressante à insérer dans le procédé de traitement s'il s'avère possible, avec les équipements industriels, d'approcher ces résultats de laboratoire.

\begin{tabular}{|c|c|c|c|c|c|c|c|}
\hline Liqueurs denses & $\%$ & \multicolumn{2}{|c|}{$Z \mathrm{n}$} & \multicolumn{2}{c|}{ Pb } \\
\cline { 3 - 8 } Produits non-flottant & Poids & Ten.(ppm) & Réc.(\%) & Ten.(ppm) & Réc.(\%) & Ten.(ppm) & Réc.(\%) \\
\hline $\mathrm{d}<2.00$ & 0.36 & 159690 & 20.84 & 115610 & 40.62 & 5609 & 27.65 \\
$2.00<\mathrm{d}>2.77$ & 99.52 & 1840 & 66.39 & 458 & 44.49 & 45 & 61.32 \\
$\mathrm{~d}>2.77$ & 0.12 & 293507 & 12.77 & 127125 & 14.89 & 6712 & 11.03 \\
\hline produit initial & 100.00 & 2758 & 100.00 & 1025 & 100.00 & 73 & 100.00 \\
\hline
\end{tabular}

Tableau 3 - Bilan de la séparation densimétrique

\subsection{Bilan des essais préliminaires de traitement}

Cette ébauche de traitement conduit actuellement à décontaminer $1 / 3$ de la boue initiale (tableau 4). Cependant, l'élimination des fines particules par cyclonage avant la flottation a écarté du procédé de traitement près de $40 \%$ de la vase initiale. Or, cette fraction granulométrique (inférieure à $10 \mu \mathrm{m}$ ) ne représente que $10 \%$ de la vase. Cela traduit que cette première coupure granulométrique effectuée avec un hydrocyclone est encore trop grossière. L'étude de la courbe de partage montre en effet une coupure à $20 \mu \mathrm{m}$. On estime que le perfectionnement de ce pré-traitement doit permettre de traiter entre 20 et $25 \%$ de boue supplémentaire. Enfin, l'optimisation de l'opération de flottation peut contribuer également à améliorer le bilan de cette opération.

\begin{tabular}{|l|r|r|r|r|r|r|r|}
\hline Fractions & \multirow{2}{*}{$\%$} & \multicolumn{2}{|c|}{ Zn } & \multicolumn{2}{|c|}{ Pb } & \multicolumn{2}{|c|}{ Cd } \\
\cline { 3 - 8 } & Poids & T.(ppm) & R.(\%) & T.(ppm) & R.(\%) & T.(ppm) & R.(\%) \\
\hline Particules $>250 \mu \mathrm{m}$ & 3.79 & 28362 & 9.30 & 23731 & 19.45 & 917 & 5.74 \\
Fines $<20 \mu \mathrm{m}$ & 37.71 & 13060 & 42.60 & 5632 & 45.93 & 604 & 37.64 \\
Concentré fottation & 24.04 & 16679 & 34.68 & 5774 & 30.02 & 1244 & 49.42 \\
Concentré densimétrique & 0.48 & 193090 & 8.02 & 11853 & 1.23 & 5878 & 4.66 \\
\hline Fraction contaminée & 66.02 & 16565 & 94.59 & 6768 & 96.63 & 893 & 97.47 \\
\hline Fraction décontaminée & 33.98 & 1840 & 5.41 & 458 & 3.37 & 45 & 2.53 \\
\hline Boue brute calculée & 100.00 & 11562 & 100.00 & 4624 & 100.00 & 605 & 100.00 \\
\hline Boue brute initiale & & 11328 & & 4924 & & 610 & \\
\hline
\end{tabular}

Tableau 4 - Bilan général du schéma de traitement, pour les trois principaux polluants

\section{Conclusion}

La boue de canal étudiée est un matériau finement divisé ( $80 \%$ des particules sont d'un diamètre inférieur à $90 \mu \mathrm{m})$ et significativement pollué $(1 \% \mathrm{Zn}, 0.5 \% \mathrm{~Pb} \ldots)$. Nous avons mis en oeuvre une méthodologie de caractérisation chimique, physique et minéralogique qui permet d'identifier la nature de la pollution. De nombreuses formes chimiques ont été déterminées. 
Nous trouvons par exemple le zinc et le plomb sous forme de sulfures, scories vitreuses, néoformations soufrées ou encore adsorbés sur des oxyhydroxydes de manganèse. La connaissance de ces formes chimiques permet d'évaluer la toxicité du produit (mobilités des polluants et risques de relargage...). Elle peut également conduire aux sources de contamination, déterminer les responsabilités du ou des pollueurs, et aider à prendre des mesures permettant d'enrayer la pollution. Enfin, elle oriente le choix des procédés de traitement.

Les essais préliminaires de décontamination de la vase étudiée conduisent à des résultats encourageants. Un schéma de procédé faisant appel aux traitements par flottation et par séparation densimétrique se révèle efficace. L'amélioration des rendements de la flottation ainsi que l'application du procédé sur une plus large gamme granulométrique permettent d'envisager très sérieusement une forte augmentation du pourcentage de boue décontaminée.

\section{Remerciements}

Cette article est la publication scientifique $\pi^{\circ} 95055$ du BRGM. Il a été financé sur un crédit de recherche du BRGM et de l'Ademe, que les auteurs tiennent à remercier.

\section{Références bibliographiques}

Barbery, G. Techniques de l'ingénieur 8-1981. Flottation : Mécanismes et réactifs.

Bovendeur, J. and Mozley, R. 1993. Characterisation and treatment of polluted material. And Environmental Application of Mineral Processing Technology - XVIII International Mineral Processing Congress. Sydney, 23-28 May 1993

Davis J.A., 1984. Complexation of trace metals by adsorbed natural organic matter. Geochimica et Cosmochimica acta vol. 48, pp.679-691.

Etcheber H., 1983. Biogéochimie de la matière organique en milieu estuarien : comportement, bilan, propriétés. Cas de la Gironde. Thèse d'état Université de Bordeaux I.

Reuter J.H., Perdue E.M., 1977. Importance of heavy metal-organic matter interactions in natural waters. Geochimica et Cosmochimica acta vol. 41, pp.325-334.

Roger B., 1988. Répartition et localisation des métaux traces dans les sédiments. Application à lestuaire de la Loire. Rapports des laboratoires. Série Environnement et génie urbain. EG-4.

Sanchez M. Etude des caractéristiques d'un matériau de Honfleur. Centre Français du littoral Laboratoire de Mécanique et Géomécanique de l'université de Nantes.

Schlosser F. 1988. Eléments de mécanique des sols. Cours de l'Ecole Nationale des Ponts et Chaussées.

Kynch, G.F. "A theory of sedimentation", Faraday society transactions, Vol. 48, 1952, pp 166176. 